\title{
Linx
}

Revue des linguistes de l'université Paris X Nanterre

9 | 1997

Émile Benveniste. Vingt ans après

\section{Portrait de la vierge en dame aux camélias}

\section{Marjolaine Hatzfeld}

\section{(2) OpenEdition \\ Journals}

Édition électronique

URL : http://journals.openedition.org/linx/1070

DOI : 10.4000/linx.1070

ISSN : 2118-9692

\section{Éditeur}

Presses universitaires de Paris Nanterre

\section{Édition imprimée}

Date de publication : 1 avril 1997

Pagination : 287-293

ISSN : 0246-8743

\section{Référence électronique}

Marjolaine Hatzfeld, " Portrait de la vierge en dame aux camélias », Linx [En ligne], 9 | 1997, mis en ligne le 09 juillet 2012, consulté le 20 avril 2019. URL : http://journals.openedition.org/linx/1070 ; DOI : 10.4000/linx.1070 


\title{
Portrait de la vierge en dame aux camélias
}

\author{
Marjolaine Hatzfeld
}

$\mathrm{D}$ u débat qui eut lieu entre linguistes et psychanalystes sur la question de l'existence, dans la langue, de mots porteurs de sens opposés, quel fut - quel est - réellement l'enjeu ? "Débat" est sans doute d'ailleurs trop dire, car quelle intersection, autre que de malentendu, peut-il y avoir entre le champ de la linguistique, qui est la langue, et le champ de la psychanalyse qui, de la langue, ne retient que ce qui fonde le sujet comme divisé par le signifiant?

Le fait de la coexistence de signifiés antithétiques accrochés à un même signifiant a quelque chose de déroutant. Comment peut-il se faire que dans la langue, lieu et origine de la différence, de toute différenciation, voire de la distinctivité comme telle - Lacan a particulièrement insisté sur la fonction du trait unaire dans la langue, "unaire" au sens du un purement différentiel, et sur sa fonction constituante pour ce qu'il appelle un "sujet" -, comment donc peutil se faire que dans la langue une même unité signifiante puisse renvoyer non seulement à des signifiés différents, mais plus radicalement à des sens opposés qui logiquement devraient s'exclure l'un l'autre? Qu'un même signifiant puisse porter à la fois et indiscernablement les signifiés de la vie et de la mort, du pur et de l'impur, du plus lointain et du plus proche, déroute l'attente que nous avons spontanément qu'à partir de la langue au moins quelque chose puisse se comprendre et être partagé distinctivement ${ }^{1}$.

Or tel est bien pourtant l'état de choses que décrivait le linguiste Carl Abel dans un article de 1884 "Sur le sens opposé des mots primitifs", et on sait que Freud crut trouver là un écho du fonctionnement que lui-même constatait dans les formations de l'inconscient. La thèse de Freud en effet, résumée, dit ceci : Les pensées refoulées attirent la parole du sujet sous la domination du

\footnotetext{
${ }^{1}$ Sur le postulat naïf du sujet parlant selon lequel l'univocité serait le régime normal de la signification, voire la très précise mise au point de M. Arrivé, Langage et psychanalyse, linguistique et inconscient, p.192.
} 


\section{Marjolaine Hatzfeld}

processus primaire. Le processus primaire, $c^{\prime}$ est une certaine façon de traiter le signe linguistique. Il isole, désolidarise, déleste le signifiant de son signifié, pour le rendre apte à être figuré en rébus sur la scène du rêve, ou à dire tout autre chose que ce qu'on était parti pour dire. L'élément sur quoi opèrent les processus dits primaires, c'est le signifiant, et rien que le signifiant, pris dans sa littéralité la plus pure, c'est à dire la plus insensée. Si Freud le premier, puis Lacan, ont isolé ainsi l'instance du signifiant de sa contrepartie de signifié, c'est qu'il s'imposait de la moindre expérience de parole (lapsus, oubli de noms, mots d'esprit), que le signifiant peut jouer sa partie de façon autonome, et se révéler après-coup support de sens inédits. C'est dans cet après coup que le sujet se constitue, d'avoir simplement pris acte du monstre linguistique qui aura franchi l'enclos de ses dents, porteur de quel sens nouveau venu là se glisser?

Or ce sens peut se présenter avec le double visage d'une tête de Janus, comme l'ont si bien nommé certains linguistes. Gegensinn, dit Abel et à sa suite Freud: "le" sens opposé, singulier fascinant par son absurdité, s'il est vrai que la relation d'opposition requiert au moins deux termes. Par ce mot unique, la langue allemande élève à la maniabilité du concept cette énigme du "deux opposés, en un". Mais qu'est ce "un", ce un de sens, dont rien ne nous permet de décider s'il penchera dans un sens ou dans l'autre ? Y aurait-il un sens audelà de la contradiction, un sens d'avant la division, d'avant le conflit? La facilité avec laquelle on fut tenté d'assigner cet un-sens-opposé à quelque état primitif de la langue témoigne de l'attrait d'un fantasme des origines où on se plaît à imaginer que la contradiction n'aurait pas encore eu cours.

Et de fait, cette présence du un-sens-opposé dans la langue du retour du refoulé ${ }^{2}$, Freud s'est demandé si elle ne serait pas - au-delà d'un phénomène qu'il pense être un phénomène régressif (qu'il ait baptisé ce processus de "primaire" le dit assez) - le propre d'un mode de pensée archaïque, qui aurait bel et bien eu cours "à l'origine", qui persisterait comme reste du passé dans ce lieu de traces que serait l'inconscient, et dont la langue même porterait ici ou là témoignage. La thèse $\mathrm{d}^{\prime} \mathrm{Abel}$ vint alors étayer pour Freud cette hypothèse génétiste ou évolutionniste, où à vrai dire se fourvoyait le sens même de sa découverte à lui, Freud. Benveniste l'a fort bien vu : Quel sens peut avoir l'idée d'un originaire qui serait, lui, au-delà du contradictoire, chez un auteur, Freud, qui le premier et contre ses contemporains, avait installé le conflit et la division au cœur du sujet ${ }^{3}$.

Il faudra donc ici distinguer deux aspects : Sur le point de savoir si ce serait un trait de primitivité de la langue que de fonctionner avec des

\footnotetext{
2 Nous évitons ici le terme d'inconscient. Est-il en effet ailleurs que là où du refoulé fait retour ? Retour d'où, de quel lieu ? Appeler ce lieu l'inconscient donne à penser qu'il existe ailleurs que dans l'articulation à en produire. Le lieu d'où est possible cette articulation serait plutôt le lieu du transfert qui couvre, le temps du déchiffrage, cette perte constituante du sujet en tant que "saisi" par le langage.

${ }^{3}$ Benveniste, E., Problèmes de linguistique générale, I, p.84.
} 
signifiants à Gegensinn, à sens-opposé, tout le monde semble d'accord, la réponse est négative. Mais sur la question plus profonde de savoir si la nature du signe linguistique comporte, ou exclut, la possibilité du Gegensinn, le débat s'obscurcit.

Pour le premier numéro de sa nouvelle revue, La Psychanalyse (1956), Lacan demanda à Benveniste de donner son point de vue sur le texte de Freud de 1910 : Sur le sens opposé des mots originaires, intitulé repris de l'article de Carl Abel. Dans ses Remarques sur la fonction du langage dans la découverte freudienne écrites à cette occasion, les critiques du linguiste peuvent se résumer ainsi : Chimérique, tant de la part d'Abel que de Freud, était l'idée d'une langue - aussi primitive qu'on la voudra, mais réelle - où un même terme dénommerait une chose et son contraire, où "tout serait soi et autre que soi, donc ni soi, ni autre". Ce qui vaut sans doute pour "les manifestations psychopathologiques du langage" n'a donc aucune valeur d'information ni ne recoupe en rien ce qu'il en est de la lange, si archaïque soit-elle.

Tout le problème est là : ces phénomènes, tératologiques pour le linguiste, que sont lapsus, calembours et autres formations de l'inconscient où pullule le Gegensinn, doivent-ils être retenus dans la réflexion sur la nature du signe linguistique, où sont-ils à évacuer comme déchets d'un dysfonctionnement ? Benveniste est là-dessus catégorique : ils ne font pas partie de la langue.

Dans le numéro suivant de la même revue (1957), par une note à son texte sur La lettre volée (que l'on trouvera dans Ecrits, p.22), Lacan, après avoir rendu hommage à "la rectification magistrale" de Benveniste, relance néanmoins le problème du sens opposé de certains mots, "primitifs ou pas". Pour lui en effet, "la question reste entière, à dégager dans sa rigueur l'instance du signifiant" Dès lors, pourquoi ce que le linguiste rejette serait-il précisément ce qui donnerait accès privilégié à ce qu'est, en toute rigueur, l'instance du signifiant ? $C^{\prime}$ est à cette provocation de Lacan que nous voudrions tenter de répondre.

Notons d'abord les deux points suivants :

$1^{\circ}$ - Les linguistes ont tranché dans leur domaine : les mots antithétiques incriminés sont en réalité des mots neutres dont la double valeur dépend du contexte, ou du point de vue d'une autre langue qui, ayant à les traduire, serait obligée d'injecter des oppositions non pertinentes dans la langue à traduire. Pour résumer avec un mot de Quine ${ }^{5}$ il n'y a "aucun principe mystique de l'identité des contraires" à invoquer pour expliquer ce phénomène. La langue, comme telle, ne se contredit pas.

$2^{\circ}$ - On peut objecter à Freud, sur son propre terrain, que le texte manifeste produit par le travail du rêve n'use pas plus de Gegensinn que la langue des linguistes. Qu'on se reporte au rêve invoqué par Freud pour sa

\footnotetext{
${ }^{4}$ C'est nous qui soulignons.

5 in Quiddités, article Etymologie.
} 


\section{Marjolaine Hatzfeld}

démonstration 6 : un seul élément, pour signifier deux idées opposées. Un seul rameau de lys, fleur de la vierge...oui, mais dont les associations ultérieures de la rêveuse disent qu'il est couvert de fleurs...rouges comme des camélias, et qui s'effeuillent. Le satisfecit que s'adresse la rêveuse sur sa conduite immaculée n'est pas sans laisser transparaître la tache de sang par quoi sa féminité rappelle ses exigences. Ce n'est donc pas un seul et même signifiant qui dit ici une chose et son contraire, mais bien la condensation spirituelle de deux signifiants distincts, dont la présence contradictoire était resté voilée dans le premier récit fait par la patiente de son rêve. Du pur à l'impur, toujours les pôles opposés nous déchirent ou nous jettent dans le ridicule : clivage du sujet entre son idéal et son désir, que le signifiant manifeste ...sans ambiguïté aucune pour qui ne recule pas devant l'aveu d'un désir qui le divise, soit devant la sorte de destitution subjective que comporte l'épreuve de l'association libre. Le sens-opposé d'un élément du rêve semble donc devoir se résoudre en toute orthodoxie linguistique, par les associations du rêveur qui tire les fils et les démêle : appel au contexte, pour faire l'appoint d'un sens. A ceci près qu'ici le contexte n'est ni dans, ni autour du texte. Qu'il soit donné par les associations libres du sujet, c'est sous la condition que celui-ci élabore, par cette parole perdue, sa propre division resté masquée, suspendue, dans le texte manifeste. Et que le sujet élabore (durcharbeitet) sa propre division ne relève d'aucune règle d'emploi, d'aucune ressource interne au système de la langue, mais d'un courage dont le "contexte" ou les coordonnées éthiques se suscitent par d'autres voies. Advenir à sa division, et ce faisant trancher le nœud du sens-opposé : ce ne sera pas l'un ou l'autre (ce qu'obtient le linguiste), ni l'un et l'autre (ce qu'obtient la synthèse dialectique ou l'au-delà mystique), mais bien l'un et l'autre dans leur irrémédiable déchirure.

Si psychanalystes et linguistes sont donc d'accord pour dissiper la "chimère" du sens-opposé, pourquoi Lacan insiste-t-il tant sur le fait que, en son fond et radicalement, le signifiant comme tel ne répond pas au principe d'identité et de non contradiction, différant de lui-même jusqu'à cette pointe extrême de l'énantiosémie?

L'analyste croit se tenir dans le droit fil de la linguistique quand il pense que le signifiant n'entraîne pas son signifié collé à la semelle de ses souliers. Leur "association" est certes posée dans les faits, et dans ce fait majeur qu'est l'existence d'un système de valeurs, mais c'est un fait également que le mouvement diachronique de la langue exige, pour être pensé, de les poser comme séparés. Or le psychanalyste accentue leur séparation plutôt que leur liaison, comme s'il s'autorisait du caractère contingent de leur association, relevé par Saussure sous le terme d'arbitraire.

En quoi, dès lors, l'analyste serait-il plus intéressé à mettre en évidence ce versant de disjonction des deux éléments du signe linguistique, plutôt que

\footnotetext{
${ }^{6}$ in L'interprétation des rêves, P.U.F p.274-5.
} 
leur liaison? C'est que cette disjonction, qui n'est pour Saussure "qu'une pure abstraction" où l'entité linguistique "s'évanouit"7, risque bien d'être pour le psychanalyste, ce qu'il y a au contraire de plus concret dans son expérience. $C^{\prime}$ est au point de déliaison du signifiant et du signifié, quand rien ne va plus dans la langue qui se met à fourcher, quand le signifiant, dans une sorte de passage à vide, ne véhicule plus aucun signifié répertorié, quand le signifié semble étrangement suspendu ou soustrait, c'est en ce point qu'un appel à prendre acte d'un nouveau sens suscite le sujet de cette faille même qui parcourt la langue. Que le signifiant qui fait ainsi entendre son appel comme non-sens, puisse être susceptible de tous les sens possibles, voire les plus contradictoires, c'est ce que l'association libre dans le cadre de l'analyse, ou le moindre mot d'esprit, manifeste amplement. Mais ce sens produit dans ce "sens dessus dessous" de la langue, par le sujet qui émerge de ce non-sens même, n'est certes pas équivalent au signifié répertorié du signe linguistique, même si il semble bien en occuper la place. Insinué à la faveur de la disjonction instantanée, fulgurante, du signifiant et du signifié, il "signifie" le sujet, ou plutôt il montre le sujet dans sa division même, ultime référent de l'articulation signifiante. C'est que le référent ici (dans l'expérience analytique) n'est pas du monde ni dans le monde. La langue n'est pas prise dans sa finalité convenue de communication - parler du monde, du monde réel, comme on prend toujours la peine de le préciser! Le référent concerne le pur rapport du sujet au signifiant, au signifiant qui l'a "causé" comme sujet en l'affectant d'une perte insensible pour prix de son inscription dans le langage. Le référent, à l'horizon de toute formation de l'inconscient, c'est cette perte qui hante celui qui est sujet "au" signifiant, et d'où s'origine tout désir.

Ce temps de disjonction du signifiant et du signifié est hors-champ de la linguistique. C'est un phénomène de sidération, de perte (perte de signifié, pour commencer par là), qu'il est convenu de mettre au compte d'une expérience "subjective". Evitons en effet le qualificatif de "psychologique", puisque c'est bien le sujet du signifiant qui à cette occasion fait surface. Mais pourquoi ne pas l'appeler, sinon linguistique, du moins langagière, cette expérience, puisque c'est la langue même qui se met à boiter, inconfortablement ?

Ce phénomène, de quelque façon qu'on veuille le qualifier, est lié à des temps où le sujet se perd, et se retrouve là où il ne se soupçonnait pas être, rivé à "une jouissance de lui-même ignorée" 8 , où il est immanquablement conduit au fil des chaînes signifiantes. Soit! Mais si une telle expérience est seulement possible - cette expérience pure de la langue, ce mouvement de roue de moulin du sens au son, puis du son au sens, où un signifiant se vide de son sens pour, autrement découpé à une lettre près, voire sensiblement le même, se charger

\footnotetext{
7 Saussure, Cours de Linguistique Générale, p.144 dans l'édition critique de Tullio de Mauro, Payot 1972.

8 On se souvient de cette expression de Freud, à propos de l'homme aux rats.
} 


\section{Marjolaine Hatzfeld}

d'un sens nouveau ou opposé - n'est-ce pas qu'elle exploite cette faille inscrite dans la structure même du signe linguistique, quel que soit l'effort du linguiste (Benveniste nommément) pour assurer une bi-univocité idéale du signifiant au signifié, qui nous conforte sur notre appartenance à un "monde" ? Mais plus la langue s'approche du "centre redoutable du désir" - en son fond désir de mort, que Lacan, en son temps", a nommé das Ding, la Chose -, plus les termes utilisés sont susceptibles de cette étrange oscillation, de ce tremblement de significations contraires, comme si l'extrême du bien pouvait se renverser dans l'extrême du mal. Phénomène de langue qui est en même temps phénomène de désir, où le désir s'égale à une défense contre le désir, phénomène de langue qui touche au plus près, au point d'en être indiscernable, au sujet réduit à cette marque d'un impossible, d'une pure division, moins entre deux termes que par rapport à la même Chose. Contradiction que la langue évite à mesure qu'elle s'éloigne du désir du sujet, de la Chose du sujet, pour parler des objets du monde. Ce que Lacan vise en plaidant pour le sens-opposé dans la langue, c'est, plus radicalement, à maintenir la fonction du signifiant, dans sa racine, comme indépendante de tout signifié, c'est à faire du signifiant un "pur signifiant", dans sa fonction d'abord de hors-sens, représentant de la Chose au-delà des significations, et plus efficace à déjouer " 1 'indistinction originelle des contraires" - à quoi le hors-sens ne peut se réduire - qu'aucune règle d'emploi de la langue. Ce signifiant hors-sens produit le sujet comme divisé par rapport à une jouissance impossible, comme désir qui ne se soutient d'aucune confusion, mais bien d'une séparation et d'une perte toujours recommencées.

Le psychanalyste, quand il reprend la définition saussurienne du signe comme purement différentiel, en insistant: pure différence à soi aussi bien, détourne-il Saussure à son usage, ou au contraire le rejoint-il au plus vif de son inlassable interrogation sur ce que peut être, dans ces conditions, l'identité du signe? De l'identité introuvable du sujet de l'inconscient à l'identité problématique du signe linguistique, du Un dans la langue au Un du sujet ${ }^{10}$, deux questionnements semblent se faire écho : ce qui n'étonnera pas, étant donné l'axiome fondamental et jamais énoncé avant Lacan, que le sujet n'est à chercher nulle part ailleurs que comme effet du signifiant, et étant donné le corrélat de cet axiome, selon lequel le signifiant est ce qui représente, et ne fait que représenter le sujet pour un autre signifiant.

"Que, sous prétexte que j'ai défini le signifiant comme ne l'a osé personne, on ne s'imagine pas que le signe ne soit pas mon affaire !" dit Lacan dans Radiophonie ${ }^{11}$. Cette audace, résumons-la dans la thèse suivante: Le langage introduit un trou dans le réel, ce réel qui, de porter la marque de la

\footnotetext{
${ }^{9}$ Séminaire de l'année 1959/60 sur L'éthique de la psychanalyse, paru au Seuil, Paris, 1986.

10 Cf. les réflexions de J.C. Milner sur le Un dans la langue, au croisement de la langue et de l'inconscient, in L'amour de la langue et Les noms indistincts, au Seuil.

11 in Scilicet 2/3, au Seuil, p. 65. Le signe ici invoqué est plutôt le signe du "pas de fumée sans feu".
} 
"coupure" signifiante, advient comme sujet au sens de Lacan. Les rapports du signifiant au sujet sont pensés comme un certain mode de couture à soi d'une surface (le sujet "sous" la trame signifiante) déchirée par cette marque, et telle que le signifiant ne peut jamais se boucler dans une identité à soi, mais toujours se divise aux abords de ce trou, provoquant ces phénomènes d'inversion, de retournement, dont l'effet de surprise signale l'appréhension de l'inconscient. Ce bizarre mode de couture à soi de cette surface, on l'aura reconnu, est une structure mœbienne tolérant, tout en ayant en chaque point un endroit et un envers, que l'on passe continûment de l'un à l'autre. Modèle qui rend compte de cette propriété du signifiant lacanien, d'être ouvert au Gegensinn, voire aux effets de sens qui culminent dans le non-sense où peut se lire, dans ce "pas-de-sens" (négation et franchissement), la présence la plus dénudée du sujet.

Il n'est pas facile, pour le psychanalyste, de cerner cette audace, d'en mesurer les conséquences, et surtout de la pratiquer. Mais la question est ici de savoir si elle renvoie à quelque chose qui pourrait n'être pas étranger aux préoccupations du linguiste. 